DESY $97-137$

December 1997

\title{
Structure Function Measurements and Kinematic Reconstruction at HERA
}

\author{
Ursula Bassler, Gregorio Bernardi \\ Laboratoire de Physique Nucléaire et des Hautes Energies \\ Université Paris 6-7, 4 Place Jussieu, 75252 Paris, France \\ e-mail: bassler@mail.desy.de; gregorio@mail.desy.de
}

\begin{abstract}
The procedure used for structure function measurements at HERA is briefly described and related to the properties of kinematic reconstruction. The reconstruction methods of the inclusive deep inelastic scattering variables are reviewed and their sensitivity to the energy and angle miscalibrations are discussed in detail. New prescriptions are introduced and related to the standard methods in order to optimize the $F_{2}$ structure function measurement over the widest kinematic range, both in the low $x$, low $Q^{2}$ and in the high $x$, high $Q^{2}$ regions. The prospects for the future high $Q^{2}$ studies are briefly discussed.
\end{abstract}




\section{Introduction}

The measurement of the structure functions of the nucleon is a major tool for the study of the strong interaction and the behaviour of the parton densities in the hadrons. To reveal possible non-standard small deviations from their well established behaviour described by the DGLAP [1] evolution equations, such as BFKL effects at low $x$ or the presence of intrinsic charm in the proton at high $x$ to name just two of them, requires a precise reconstruction of the deep inelastic scattering (DIS) kinematics over the widest possible kinematic range. With the advent of the HERA electron-proton collider, this reconstruction no longer needs to rely on the scattered lepton only, since the most important part of the hadronic system is visible in the almost hermetic H1 and ZEUS detectors. This redundancy allows for an experimental control of the systematic errors and of the radiative corrections to the structure function measurement if it is based on several independent methods to determine the usual DIS kinematic variables $x, y, Q^{2}$ :

$$
x=\frac{Q^{2}}{2 P . q} \quad y=\frac{P . q}{P . k} \quad Q^{2}=-\left(k-k^{\prime}\right)^{2}=-q^{2}=x y s
$$

with $s$ being the ep center of mass energy squared, $P, k$ the 4-vectors of the incident proton and lepton, and $k^{\prime}$ of the scattered lepton. Since many different reconstruction methods have already been used at HERA, it is a natural objective to optimize this reconstruction, and to try to find "the best" method, or at least to justify the use of a given method instead of a kinematic fitting procedure for instance. In this report we briefly sketch in section 2 the procedure used so far to measure $F_{2}$ at HERA, and relate it to the effects of kinematic reconstruction. In section 3 we review the methods of kinematic reconstruction used at HERA, and we classify them on the basis of their properties. In section 4 we discuss possible improvements of these reconstruction methods, in particular those related to the study of low $x$ physics. In section 5 we study the effect of the hadronic final state and scattered electron reconstruction errors on the kinematic methods in order to understand the choices made in this field by the two HERA collaborations. In section 6 , the high $Q^{2}$ case is treated in more detail due to its future importance and also since there are new possibilities in this kinematic regime. In conclusion, we briefly provide some prospects on the influence of these technical matters on the structure function program of the next decade.

\section{Structure Function Measurements}

The cross-section for the DIS reaction $e^{+}+p \rightarrow e^{+}+X$ with unpolarized beams is:

$$
\frac{d^{2} \sigma}{d x d Q^{2}}=\frac{2 \pi \alpha^{2}}{x Q^{4}}\left[Y_{+} F_{2}\left(x, Q^{2}\right)-y^{2} F_{L}\left(x, Q^{2}\right)-Y_{-} x F_{3}\left(x, Q^{2}\right)\right]\left(1+\delta_{r}\left(x, Q^{2}\right)\right)
$$

In this equation $\alpha$ is the electromagnetic coupling, $F_{2}$ is the generalized structure function which reflects both photon and $Z^{\circ}$ exchange, $F_{L}$ is the longitudinal structure 
function, $F_{3}$ is the parity violating term arising only from the $Z^{\circ}$ exchange, $\delta_{r}$ is the electroweak radiative correction. The helicity dependence of electroweak interactions, is contained in the functions $Y_{ \pm}(y)=1 \pm(1-y)^{2}$.

At HERA, these three structure functions of the proton can be measured, in particular $F_{2}$ which has already been measured over several orders of magnitude in $x$ and $Q^{2}$ with a precision of about $5 \%$. Although apparently only one observable $\left(\frac{d^{2} \sigma}{d x d Q^{2}}\right)$ is related to the three structure functions, the problem can be solved because in some kinematic region only one of them has a relevant contribution and/or because the beam conditions (energy, lepton sign) can be changed, hence changing the coefficient in front of each of them, for a given $\frac{d^{2} \sigma}{d x d Q^{2}}$. In the following, we will not study the procedure to derive them from the cross-section measurement (which are described in [3] for instance), but concentrate on the determination of $\frac{d^{2} \sigma}{d x d Q^{2}}$.

Generally, the value of $\frac{d^{2} \sigma}{d x d Q^{2}}$ at the point $\left(x_{o}, Q_{o}^{2}\right)$ is experimentally determined by the number $N_{\Delta}^{D}$ ( " $D$ " stands for Data) of DIS events observed in an $\left(x, Q^{2}\right)$ bin $\Delta$ centered around $\left(x_{o}, Q_{o}^{2}\right)$, normalized to the integrated luminosity $L^{D}$ accumulated during the data taking and corrected by an acceptance factor $T_{\Delta}^{D}$ which depends on the event selection cuts and on the detector response:

$$
\frac{d^{2} \sigma}{d x d Q^{2}}=\frac{C_{\Delta}}{L^{D}} \frac{N_{\Delta}^{D}}{T_{\Delta}^{D}}
$$

$C_{\Delta}$ is a numerical factor which takes into account the surface of the bin. Actually, resolution effects cause migration of events from one bin to other bins, so the previous relation should be treated in fact as a matrix relation, with the cross-section being related to all bins via the inverse of a matrix $T(i j)$, in which every matrix element $(i j)$ gives the probability that an event originating from the bin $i$ is reconstructed in a bin $j$. Thus the double differential cross-section measured at the center of the bin $(i)$ is:

$$
\frac{d^{2} \sigma}{d x d Q^{2}}=\frac{C_{\Delta}}{L^{D}} \sum_{j} N_{j}^{D} T^{-1}(i j)
$$

The matrix $T$ can be obtained by simulating a large sample of DIS events, provided the simulation is able to reproduce the detector effects. However to solve equation (4) one needs to invert this matrix. This is a non-trivial task due in particular to numerical instabilities [ [4, 5, 6], which has not been done yet for the published HERA structure function measurements. Instead, an iterative procedure is used to solve equation (3). Obviously, if the structure function used in the simulation is equal to the one to be measured, and assuming that $T_{\Delta}^{D}=T_{\Delta}^{S}$ (" $S$ " stands for simulation), we have:

$$
\frac{d^{2} \sigma}{d x d Q^{2}}=\frac{N_{\Delta}^{D}}{L^{D}} \frac{L^{S}}{N_{\Delta}^{S}} \frac{d^{2} \sigma^{S}}{d x d Q^{2}}
$$

This equation is solved iteratively, starting the simulation from a "guessed" structure function parametrization, and replacing it at each iteration by the parametrization obtained from a fit to the structure function "measured" at the previous iteration. 
In three or four iterations the result is stable within one $\%$ or less (see [7] for a more detailed discussion) in the measureable regions. These measureable regions are empirically characterized by values of $T_{\Delta}$ close to unity. In order to define them in a more rigorous way and to understand their relation to the kinematic reconstruction, we will now study the $T_{\Delta}$ factors.

We distinguish two ways of defining the bin $\Delta$ : one $(\Delta, t)$ based on the "true" kinematic variables, defined at the hadronic vertex; the other $(\Delta, r)$ based on the reconstructed variables. We also consider the effect of the "event selection cuts" on the number of events $\left(N_{\Delta}^{c}\right)$ compared to the number of events before the cuts $\left(N_{\Delta}\right)$. These "cuts" are imposed to improve the precision of the measurement, but can have an influence on the distribution of the events in the kinematic plane, so the two cases must clearly be separated. With these definitions, we deduce from eq. (3) that $T_{\Delta}$ can be expressed as [8]:

$$
T_{\Delta}=\frac{N_{\Delta, r}^{c}}{N_{\Delta, t}}=\epsilon_{\Delta, t} \cdot A_{\Delta} \quad \text { with } \quad \epsilon_{\Delta, t} \equiv \frac{N_{\Delta, t}^{c}}{N_{\Delta, t}} \quad \text { and } \quad A_{\Delta} \equiv \frac{N_{\Delta, r}^{c}}{N_{\Delta, t}^{c}}
$$

The first term $\left(\epsilon_{\Delta, t}\right)$ characterizes the "efficiency" of the cuts in the bin $\Delta$, while the second one can be defined as the "smearing acceptance" of the bin $\Delta$, since only the smearing of the kinematic reconstruction is involved in its variations. Since the cuts are choosen to have a high efficiency, we will not discuss here the difference at the percent level which might occur between $\epsilon_{\Delta}$ as determined on the data and on the simulation, but focus on the behaviour of the smearing acceptance, which can have large variations (up to hundreds of percents) across the kinematic plane, hence determining the measureable regions. The smearing acceptance variations are better studied considering the $(\Delta, i)$ subset which contains the events belonging to $(\Delta, i)$ and $(\Delta, r)$. Then $A_{\Delta}$ can be rewritten as

$$
A_{\Delta}=\frac{S_{\Delta}}{P_{\Delta}} \quad \text { with } \quad S_{\Delta} \equiv \frac{N_{\Delta, i}^{c}}{N_{\Delta, t}^{c}} \quad \text { and } \quad P_{\Delta} \equiv \frac{N_{\Delta, i}^{c}}{N_{\Delta, r}^{c}} \quad ; \quad N_{\Delta, i} \equiv N_{\Delta, t \cap \Delta, r}
$$

The $S_{\Delta}$ and $P_{\Delta}$ are refered as the stability and the purity of the bin $\Delta$, since they characterize respectively:

- the proportion of genuine events of a bin, which are reconstructed in the same bin. $S_{\Delta}$ characterizes the number of events which migrate outside of the bin $\Delta$.

- the ratio of genuine events of a bin reconstructed in the same bin divided by the total number of events reconstructed in that bin. This last number is influenced by the number of events originating from other bins which migrate into $\Delta$.

The obvious goal is to maintain a stability and a purity as close to unity as possible, hence $A_{\Delta}$ will also be close to 1 . However $A_{\Delta}$ can be close to unity even for a low stability bin, if its purity roughly matches its stability. In order to ensure a reliable measurement of the cross-section, we will thus enforce conditions on $S_{\Delta}$ and $P_{\Delta}$ separately, rather that only on their ratio. To make a concrete example let us examine 
the three standard reconstruction methods used for DIS neutral currents at HERA, in the $\left(x, Q^{2}\right)$ binning used by the H1 collaboration, i.e 8 (5) bins per order of magnitude in $Q^{2}(x)$ 99. The smearing acceptance, stability and purity for the H1 detector are shown in fig. 11 for the electron, Double-Angle [10 and $\Sigma$ [11 methods, in $x$ bins, at $Q^{2}$ $=20 \mathrm{GeV}^{2}$. As mentioned, the acceptance can be equal to unity, while the stability
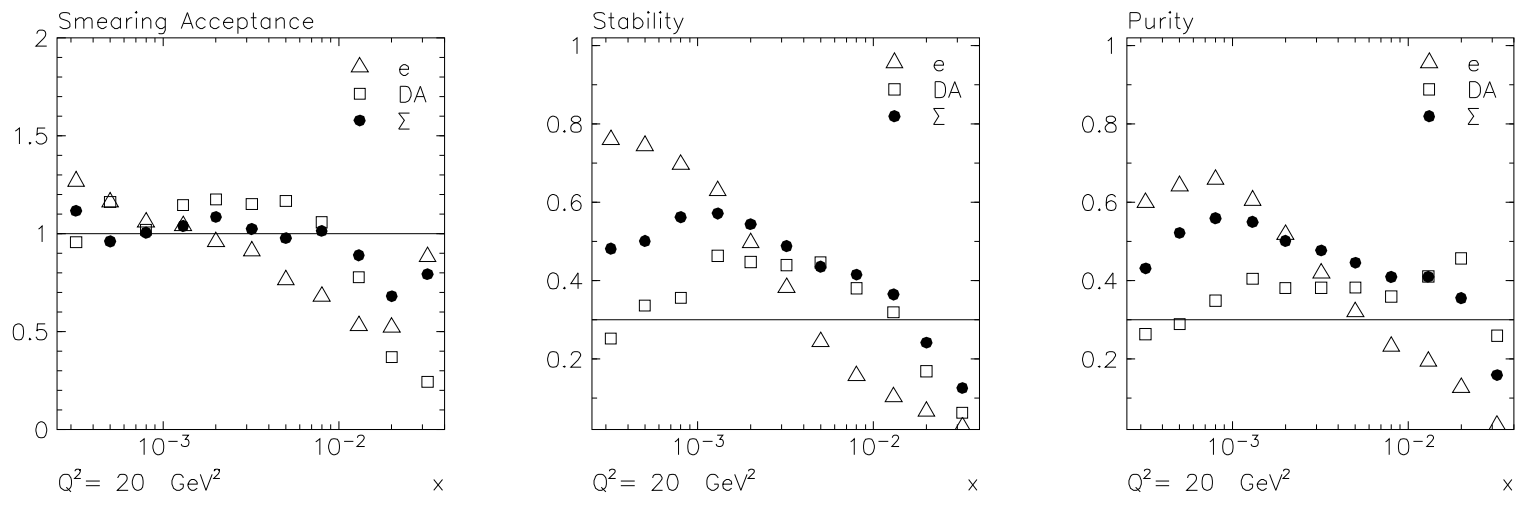

Figure 1: Smearing Acceptance, Stability and Purity at $Q^{2}=20 \mathrm{GeV}^{2}$ for the 3 kinematic reconstruction methods: e (open triangles), DA (open squares), $\Sigma$ (closed circles).

and the purity are low $(\sim 25 \%)$, like for instance at the lowest $x$ point in the DA method. Conversely, by requiring a "reasonably" high stability and purity (we will use throughout this paper a minimum of $30 \%$, as the $\mathrm{H} 1$ collaboration did for this binning [9]), we ensure an acceptance close to unity.

We observe in fig. 1 that the stability has a more regular behaviour than the purity or the acceptance, since it reflects only the combination of the resolutions in $x$ and $Q^{2}$ in a given bin. The stability can be increased by enlarging the size of the bin. Although the stability and purity are related quantities (they share the same numerator), the purity is more irregular because it is influenced by events coming from other bins, which may be populated in a different way, and have different resolutions in the kinematic variables. Like the stability, the purity can also be increased by enlarging the bin size, however the migrations inside the bin depend on several factors (resolutions in different bins, population of different bins, structure function values in regions which are not "measurable", radiative effects), rendering the control of the purity more delicate than the stability. Thus, in the following, when comparing different methods, we will make the comparison on their purity, but only in the bins having a minimum stability (chosen to be $30 \%)$.

\section{Kinematic Reconstruction Methods at HERA}

In the naive quark-parton model (QPM), the lepton scatters elastically with a quark of the proton, and the two body final state is completely constrained using two variables, 
if we know the initial energies labeled $\mathrm{E}_{\circ}$ and $\mathrm{P}_{\circ}$ of the electron and proton. Similarly, the DIS variables can be determined using 2 independent variables, which can be the energy $(\mathrm{E})$ of the scattered electron, its polar angle円 $(\theta)$ or independent quantities reconstructed out of the hadronic final state particles. For instance $\Sigma$, obtained as the sum of the scalar quantities $E_{h}-p_{z, h}$ of each particle (assumed to be massless) belonging to the hadronic final state, $p_{T, h}$ as its total transverse momentum or the inclusive angle $\gamma$ of the hadronic system[ which corresponds to the angle of the scattered quark in the QPM:

$$
\Sigma=\sum_{h}\left(E_{h}-p_{z, h}\right) \quad p_{T, h}=\sqrt{\left(\sum_{h} p_{x, h}\right)^{2}+\left(\sum_{h} p_{y, h}\right)^{2}} \quad \tan \frac{\gamma}{2}=\frac{\Sigma}{p_{T, h}}
$$

$E_{h}, p_{x, h}, p_{y, h}, p_{z, h}$ are the four-momentum vector components of each hadronic final state particle. $\Sigma$ is by construction minimally affected by the losses in the forward direction due to the beam pipe hole in which the target jet and the initial state gluon radiation tend to disappear. $p_{T, h}$ covers the other spatial dimensions, and is more sensitive to forward losses. Thus to improve the kinematic reconstruction we should avoid using $p_{T, h}$ directly. We can use $\gamma$ instead, which carries the $p_{T, h}$ information and is better measured since in the ratio $\Sigma / p_{T, h}$ the energy uncertainties cancel to first order and the effect of the losses in the forward beam pipe is diminished. Thus the optimal four "detector oriented" variables to characterize deep inelastic scattering at HERA are $[\mathrm{E}, \theta, \Sigma, \gamma]$.

Using these four input variables, there are three basic methods which make use of only two of them at a time, and which are precise enough to allow sensible kinematic reconstruction, namely:

- The electron only method $(e)$ which uses $\mathrm{E}$ and $\theta$.

- The hadrons only method $(h)$ which uses $\Sigma$ and $\gamma$ 12]

- The double angle method (DA) which uses $\theta$ and $\gamma[10$.

In the following, all methods (for instance the DA) using some information from the hadronic final state will be called "hadronic", although strictly speaking there is only one inclusive hadronic method (a complete set of formulae is given in the appendix). The $h$ method will not be discussed in the following since it is not precise enough compared to the others, for neutral current DIS events.

More than two variables are needed to determine the kinematics if the incident energy is unknown, which is the case when the incident electron emits a photon before the hard collision. This photon is often undetected since it is emitted colinearly with the incident electron beam direction, and can thus escapes inside the beam pipe. In this case three variables are needed to reconstruct the kinematics. For instance $y_{h}=\Sigma / 2 E_{\text {。 }}$ can be replaced by $y_{\Sigma} \equiv \Sigma /\left(\Sigma+\Sigma_{e}\right)$ to take into account the missing energy due to the escaping photon. A further important characteristic of $y_{\Sigma}$ is that at high $y$,

\footnotetext{
${ }^{1}$ The positive $z$ axis is defined at HERA as the incident proton beam direction.

${ }^{2}$ We can define the similar quantities for the scattered electron:

$$
\Sigma_{e}=E(1-\cos \theta) \quad p_{T, e}=E \sin \theta \quad \text { i.e. } \tan \frac{\theta}{2}=\frac{\Sigma_{e}}{p_{T, e}} .
$$
}


$\Sigma$ becomes the dominant term in the denominator and thus experimental errors on the $\Sigma$ measurement tend to cancel between numerator and denominator. In the $\Sigma$ method [11], $Q_{\Sigma}^{2}$ is constructed, like $y_{\Sigma}$ to be independent of QED initial state radiation (ISR) and to be optimal in terms of resolution; thus $p_{T, e}$ is used instead of $p_{T, h}$ :

$$
Q_{\Sigma}^{2} \equiv p_{T, e}^{2} /\left(1-y_{\Sigma}\right) \quad x_{\Sigma} \equiv Q_{\Sigma}^{2} / s y_{\Sigma}
$$

Insensitivity to ISR on $x_{\Sigma}$ is achieved simply by replacing $s$ by $2 \mathrm{P}_{\circ}\left(\Sigma+\Sigma_{e}\right)$, thereby obtaining the I $\Sigma$ method which is based on $(\mathrm{E}, \theta, \Sigma)$. The DA method was also rendered ISR independent by using $\mathrm{E}$ to reconstruct the initial electron beam energy. The IDA method [10] obtained in this way is thus based on $(\mathrm{E}, \theta, \gamma)$. The complete formulae and the comparison of these two methods can be found in 11$]$. They will not be considered in the following, since the gain obtained by the complete independence to ISR is not sufficient to compensate the loss of precision induced by the reconstruction of the incident electron energy.

Fig. 2 shows the purity of the $e$, DA and $\Sigma$ methods as a function of $x$ in bins of $Q^{2}$. The properties of the 3 standard methods to reconstruct the kinematics of neutral current DIS events at HERA are clearly visible: high precision of the $e$ method at high $y$ with a severe degradation at low $y$, good precision for the $\Sigma$ method in the complete kinematic range, high precision of the DA method at medium $\left(\sim 10^{2} \mathrm{GeV}^{2}\right)$ and high
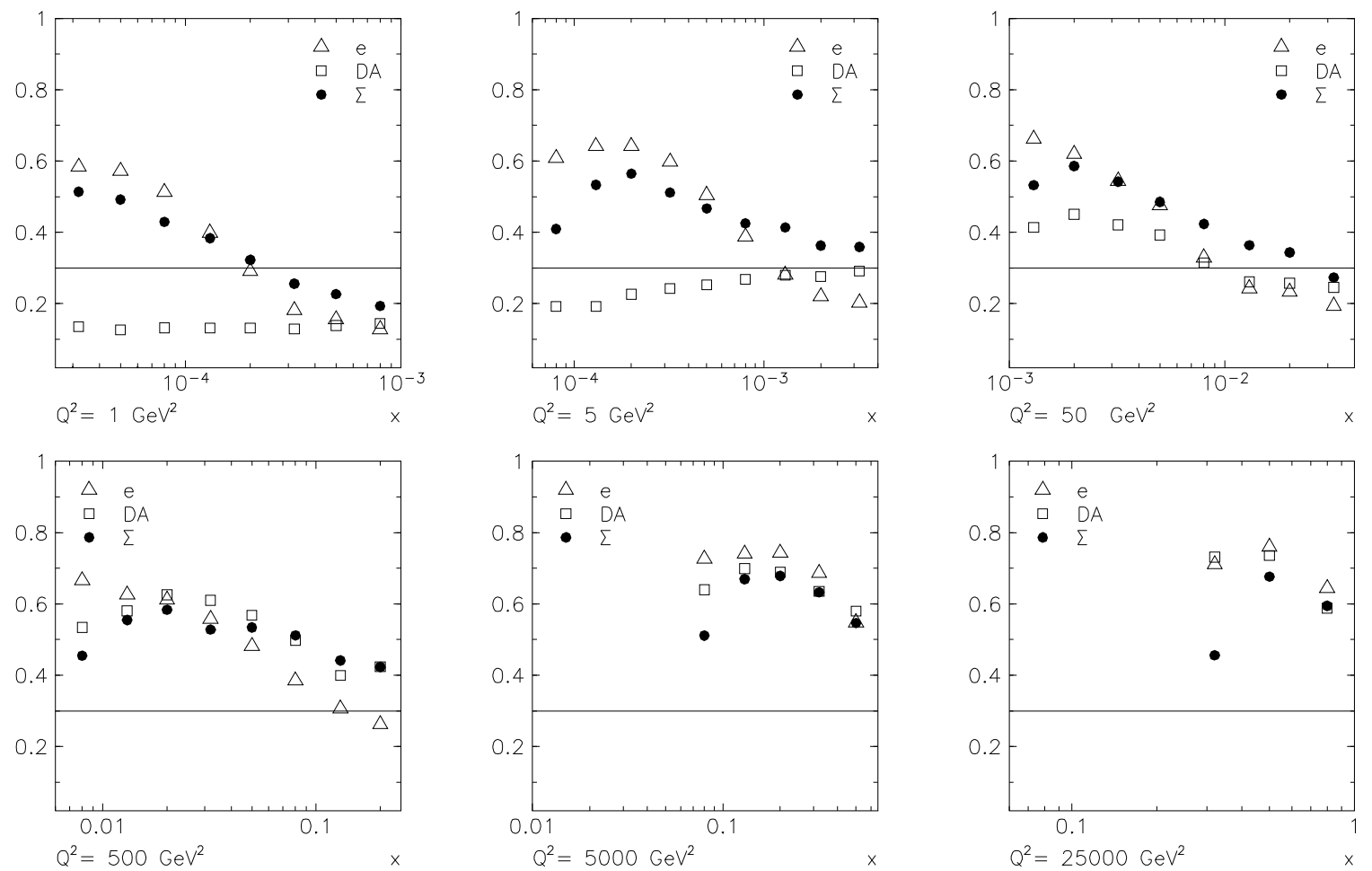

Figure 2: Purity at $Q^{2}=1,5,50,500,5000$ and $25000 \mathrm{GeV}^{2}$ for the 3 kinematic reconstruction methods: e (open triangles), DA (open squares), $\Sigma$ (closed circles). 
$Q^{2}\left(\sim 10^{4} \mathrm{GeV}^{2}\right)$ with a severe degradation at low $Q^{2}\left(\sim 1 \mathrm{GeV}^{2}\right)$.

The "hadronic" methods have already been shown to display at low $x$ and low $Q^{2}$ a rather imprecise reconstruction of $Q^{2}$. A simple solution to this problem is to use $Q_{e}^{2}$ and to obtain, via $Q^{2}=x y s$, either $x$ from a hadronic $y$ or $y$ from a hadronic $x$. For instance we have the mixed method [13] $\left(x_{m}\right.$ is obtained from $y_{h}$ and $\left.Q_{e}^{2}\right)$ which has a good precision at low $y$ [14, 15]. The precision at high $y$ can be improved by using the mixed $\Sigma\left(m \Sigma ; y_{m \Sigma} \equiv y_{\Sigma}\right.$ and $\left.Q_{m \Sigma}^{2} \equiv Q_{e}^{2}\right)$, or better the $e \Sigma \operatorname{method}\left(x_{e \Sigma} \equiv x_{\Sigma}\right.$ and $\left.Q_{e \Sigma}^{2} \equiv Q_{e}^{2}\right)$ as was shown on fig. 2 and 3 of ref. [11].

Another approach to combining two methods which have complementary properties has been tried by the $\mathrm{H} 1$ collaboration in the analysis of the diffractive structure function [16]. Since $Q^{2}=4 \mathrm{E}_{\circ}^{2}(1-y) / \tan ^{2} \frac{\theta}{2}$ both in the $e$ and DA methods, an "average" method (labeled here ADA) has been introduced, in which $y_{A D A}$ is obtained by weighting $y_{e}$ and $y_{D A}$ by $y$ and $(1-y)$ respectively:

$$
y_{A D A} \equiv y_{e}^{2}+y_{D A}\left(1-y_{D A}\right) \quad Q_{A D A}^{2} \equiv \frac{4 E_{\circ}^{2}\left(1-y_{A D A}\right)}{\tan ^{2} \frac{\theta}{2}} .
$$

On the non-diffractive DIS events a better complementarity is actually achieved in the A $\Sigma$ method by "averaging" the $e$ and the $\Sigma$ methods [17]. However neither the A $\Sigma$ nor the ADA method bring an improvement compared to the simpler $e \Sigma$ method as can be seen in fig. 3: the $\mathrm{A} \Sigma$ method is giving similar performances to the $e \Sigma$ one except at low $x$ where it is slightly less precise, while the ADA method is better at high $x$ and high $Q^{2}$, but weaker elsewhere, in particular at low $Q^{2}$.
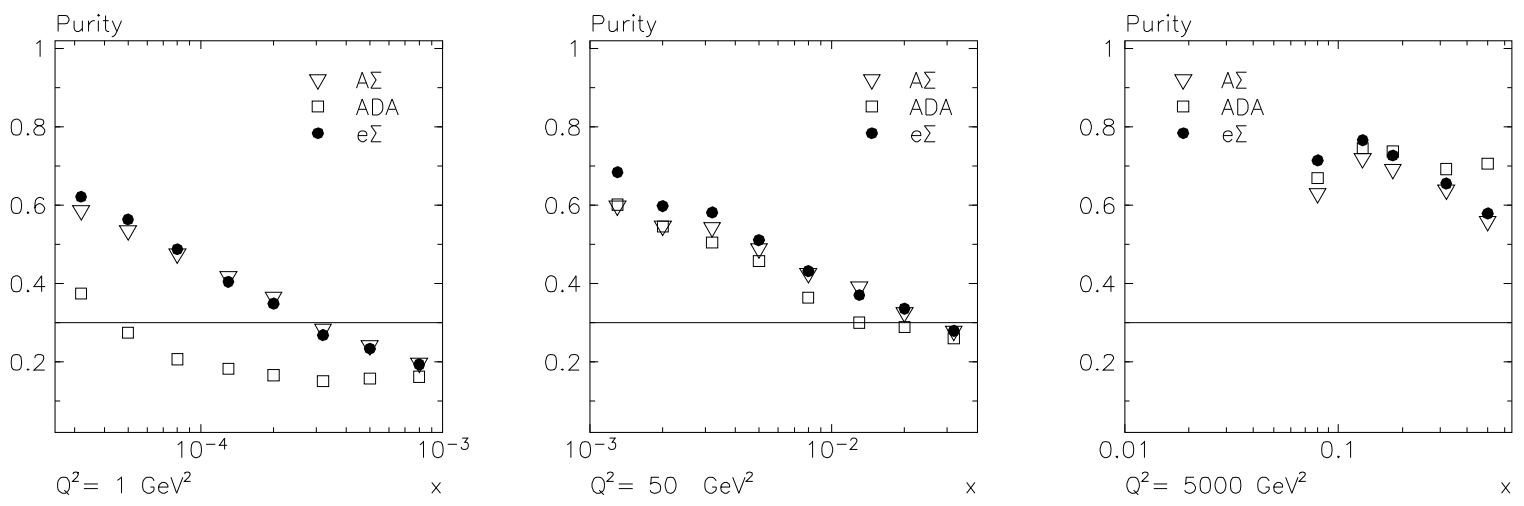

Figure 3: Purity at $Q^{2}=1,50$ and $5000 \mathrm{GeV}^{2}$ for the 3 kinematic reconstruction methods: $A \Sigma$ (open triangles), ADA (open squares), $e \Sigma$ (closed circles).

Before ordering these methods to underline their relations, let us introduce another method derived from the DA method, which will be useful later, for the understanding of more complicated methods and for the study of error propagation. Following the logic used for the $\Sigma$ method, the inclusive hadronic angle $\gamma$ can be replaced by $\gamma_{\Sigma}$ defined by $\tan \gamma_{\Sigma} / 2=\Sigma / p_{T, e}$ since transverse momentum conservation implies $p_{T, h}=$ 
$p_{T, e}$ even in the case of colinear ISR. This replacement improves the precision on the hadronic angle but has the obvious drawback of introducing a sensitivity to the electron energy reconstruction errors, which is absent in the DA method. This D $\Sigma$ method defined using $\gamma_{\Sigma}$ instead of $\gamma$ in the DA formulae, gives:

$$
y_{D \Sigma}=\frac{\tan \gamma_{\Sigma} / 2}{\tan \gamma_{\Sigma} / 2+\tan \theta / 2}=\frac{\Sigma / p_{T, e}}{\Sigma / p_{T, e}+\Sigma_{e} / p_{T, e}}=y_{\Sigma} .
$$

The $Q_{D \Sigma}^{2}$ and $x_{D \Sigma}$ are nevertheless different from their $\Sigma$ counterparts (although $x_{D \Sigma}=$ $x_{m}$ ) and we will see in the section 5 how the hadronic and electron miscalibrations affect them.

In fig. 1 the distribution of the reconstructed $x\left(x_{r e c}\right)$ divided by the true $x\left(x_{g e n}\right)$ and the equivalent distribution for $Q^{2}$ are compared for the $e, \mathrm{DA}, \Sigma, \mathrm{D} \Sigma$ methods at high $y(0.3-0.7)^{3}$. This figure, as all the figures in this paper, is obtained from a deep

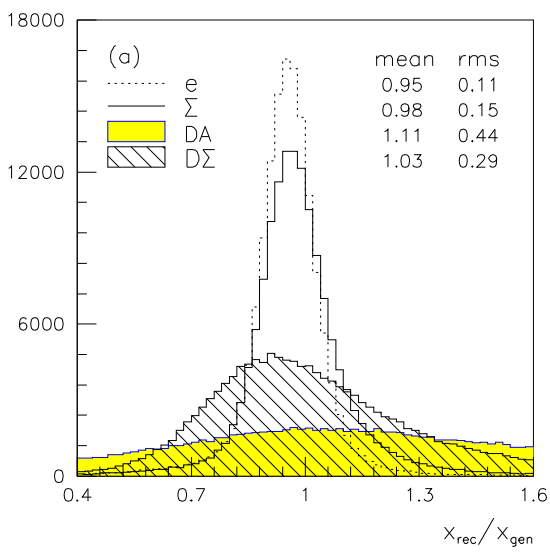

Figure 4: Distribution of $x_{r e c} / x_{\text {gen }}$ (a) and $Q_{r e c}^{2} / Q_{\text {gen }}^{2}$ (b) for the e, DA, $\Sigma$ and $D \Sigma$ methods for $Q^{2}>7 \mathrm{GeV}^{2}$ at high $y(0.3<y<0.7)$

inelastic sample simulated in detail in the $\mathrm{H} 1$ detector (1995 set-up) as described in 18. All known effects both from the detector and from the physics point of view (structure functions, QED radiation) are included in the simulation, which has been shown to give a good description of the $\mathrm{H} 1$ data in the complete kinematic range [18, 19, 20. The uncertainty on the electron energy, on the polar angle and on the hadronic energy scale are typically 1\%, $1 \mathrm{mrad}$ and $4 \%$ respectively. For figs. 4 and 5 , a cut at $Q^{2} \geq 7$ $\mathrm{GeV}^{2}$ has been made, which corresponds at HERA to the region measured with the highest precision. In fig. 4 the excellent resolution at low $x$ and low $Q^{2}$ of the $e$ method is clearly visible. Only the $\Sigma$ method has a comparable, although lower, resolution in $x$. In $Q^{2}$ the weakness of all these hadronic methods is visible. The $\mathrm{D} \Sigma$ method is more precise than the DA one at low $Q^{2}$, as expected from the way it is constructed. Unfortunately the $e$ method is not precise at low $y$ (as can be seen in fig. 2), so either the

\footnotetext{
3 At low $y$, since all hadronic methods give rather similar results in terms of resolution, (contrary to the $e$ method which strongly degrades), the comparison is better made using the plots of the purity (fig. 2). The purity of the $\mathrm{D} \Sigma$ method is almost identical to the $\Sigma$ one at low $y$ (not shown).
} 


\begin{tabular}{|c|c|c|c|}
\hline & Sigma & Double-Angle & Mixed \\
\hline basic method & $h$ & $\mathrm{DA}(\gamma)$ & $m$ \\
\hline ISR independent & $\mathrm{I} \Sigma$ & $\mathrm{IDA}(\gamma)$ & $m \Sigma$ \\
\hline optimized method & $\Sigma$ & $\mathrm{D} \Sigma\left(\gamma_{\Sigma}\right)$ & $e \Sigma$ \\
\hline \hline rescaled method & $r \Sigma$ & $\mathrm{PT}\left(\gamma_{r \Sigma}\right)$ & $r e \Sigma$ \\
\hline
\end{tabular}

Table 1: Classification of the hadronic methods. For the methods using the DoubleAngle approach, the correponding hadronic angle is also given. Note that the $m \Sigma$ method is ISR independent only in $y$. The methods displayed in the last row are discussed in section 4.

structure function measurement is done using different methods in different regions of the kinematic plane, or a precise method over the complete kinematic plane is found. Both these approaches have already been used at HERA, but for the more precise future measurements it is mandatory to focus on the second case and to optimize the reconstruction method to be used.

To conclude this section, the hadronic methods are ordered in table 1: each column represents the type of approach followed (Sigma, Double-Angle, Mixed), while each row represents a prescription to derive them: basic method, ISR independent, "optimized resolution". The relations become clear and the effects coming from the type of recontruction used can be disentangled, by comparing methods from the same row, from those due to the detector response which can be studied by comparing methods from the same column.

\section{Kinematic Reconstruction Improvements}

The description of the methods given above suggests that it might be possible to further optimize the reconstruction precision. The most direct way would be to perform a fit of the kinematics using all information available as described for instance in [21, 22]. However the redundancy is not large, since $p_{T, h}$ is not precise enough to be helpful in neutral current events, except at high $Q^{2}$. Furthermore the method requires a good knowledge of the uncertainties of the measured variables over all the kinematic plane and the lack of statistics has prevented the production of a detailed enough map of all imperfections of the detectors. We will thus concentrate on the "analytic" improvement of the hadronic methods, in particular by "rescaling" the hadronic energy: $\Sigma$ may be rescaled to approach its true value using the formula $\Sigma+\Sigma_{e}=2 \mathrm{E}_{\circ}$, if the error on $\Sigma_{e}$ 
is assumed to be small compared to the $\Sigma$ one 1 . But if $\Sigma$ is expressed as $2 \mathrm{E}_{\circ}-\Sigma_{e}$ in the $h$ or $\Sigma$ method, these methods become identical to the $e$ method. An intermediate solution between the $\Sigma$ and $e$ method can be obtained by using the rescaling factor defined as

$$
r \equiv \frac{2 E_{\circ}}{\Sigma+\Sigma_{e}}
$$

as was already implicitly done in some of the previously discussed variables:

$$
y_{\Sigma}=r y_{h} ; \quad y_{e \Sigma}=r y_{\Sigma} ; \quad Q_{\Sigma}^{2}=\frac{Q_{e}^{2}}{r} ; \quad Q_{D \Sigma}^{2}=Q_{e}^{2} r
$$

An improvement on the $\Sigma$ kinematic variables at low $x$ is obtained by directly rescaling $\Sigma$, thereby defining an $r \Sigma$ method:

$$
y_{r \Sigma} \equiv \frac{r \Sigma}{r \Sigma+\Sigma_{e}} \quad Q_{r \Sigma}^{2} \equiv \frac{p_{T, e}^{2}}{1-y_{r \Sigma}}
$$

The corresponding "rescaled" hadronic angle is defined as $\tan \gamma_{r \Sigma}=r \Sigma / p_{T, e}$ and allows to define a rescaled $\mathrm{D} \Sigma$ method $(r \mathrm{D} \Sigma)$. This method was already derived in a different way by the ZEUS collaboration, and is called the "PT" method [7]. Similarly an $r e \Sigma$ method is defined, using $x_{r \Sigma}$ and $Q_{e}^{2}$. With these definitions we create the last row of table 11 with the "rescaled" methods. These new methods are compared to the $e$ and $\Sigma$ methods in $x$ and $Q^{2}$ in fig. 5 in the same $Q^{2}, y$ intervals as those of fig. 4 . With
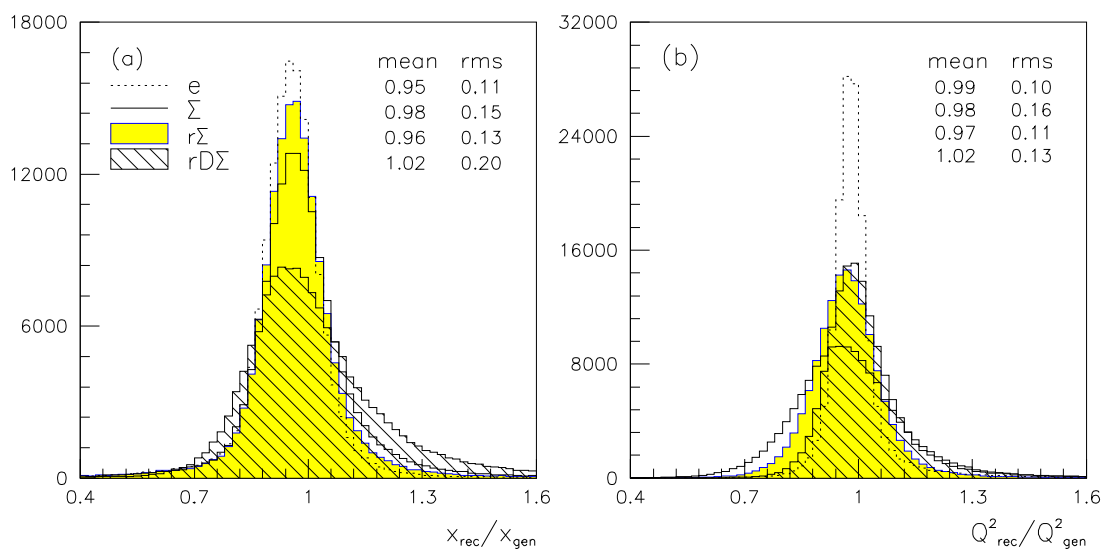

Figure 5: Distribution of $x_{\text {method }} / x_{\text {gen }}$ (a) and $Q_{\text {method }}^{2} / Q_{\text {gen }}^{2}$ (b) for the e, $\Sigma, r \Sigma$ and $r D \Sigma(\equiv P T)$ methods for $Q^{2}>7 G e V^{2}$ at high y $(0.3<y<0.7)$.

the rescaling, $x_{P T}\left(x_{r \Sigma}\right)$ has indeed a better resolution than $x_{D \Sigma}\left(x_{\Sigma}\right)$. However $x_{P T}$ is still less precise for $y$ values between 0.3 and 0.7 . than the simple, non-rescaled, $x_{\Sigma}$, due to the propagation of the hadronic error in these two methods (cf section 5). The

\footnotetext{
${ }^{4}$ Indeed, the relative error on $\Sigma$ increases when going to lower $x$ and lower $Q^{2}$ since the hadronic final state contains an increasing fraction of low energy particles which are measured less precisely and which may go undetected in the HERA detectors.

${ }^{5}$ Actually, the "PT" method includes also a calibration of the hadronic final state using $p_{T, e}$. Here we single out the analytic definition of the method.
} 
difference in $x$ between the rescaled methods and the $e$ method is now smaller, which is also true in $Q^{2}$ where the rescaled methods $(r \Sigma$ and PT) are similar and definitely better than the non-rescaled ones $(\Sigma$ and $\mathrm{D} \Sigma)$. By using the $Q_{e}^{2}$, the $\operatorname{re} \Sigma$ combination allows a further improvement with respect to the two other rescaled methods or to the $e \Sigma$ method. The overall behaviour in the complete kinematic plane of one of the rescaled methods (the $\mathrm{PT}$ ), compared to the $e \Sigma$ can be judged by their purity shown in fig. 6. They both show a precise behaviour over the complete kinematic plane. The $e \Sigma$ method is more precise at low $x$, at high $Q^{2}$, and has a comparable or better purity than the $e$ method (compare fig. 2 and fig. 6) even at low $x$ and low $Q^{2}$.
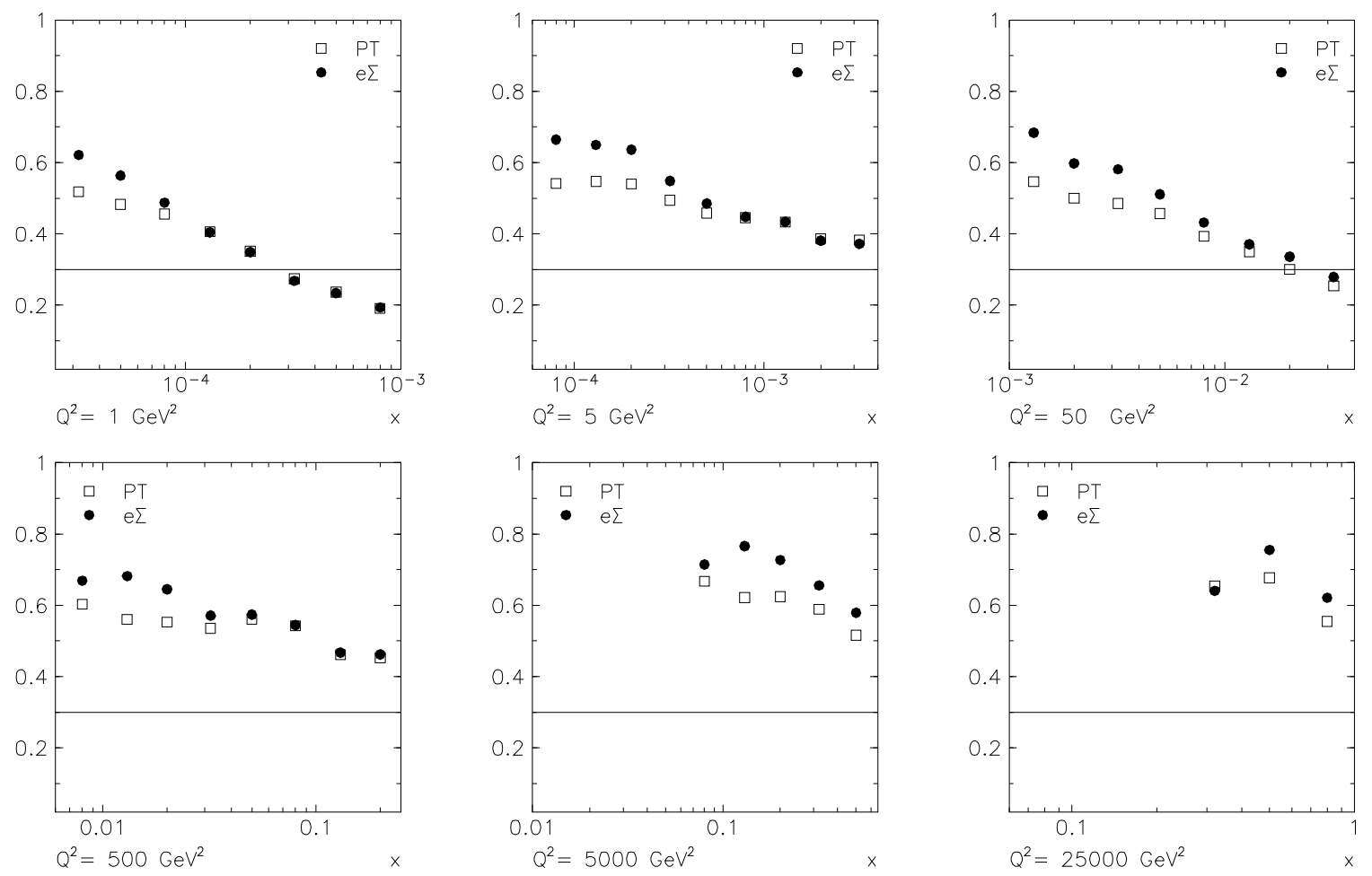

Figure 6: Purity at $Q^{2}=1,5,50,500,5000$ and $25000 \mathrm{GeV}^{2}$ for the PT (open squares) and $e \Sigma$ (closed circles) kinematic reconstruction methods. With the high precision measurement of the electron energy in the H1 detector, the $e \Sigma$ method is better than the PT method at low $x$ and also at high $Q^{2}$ (see fig. 7 for further comments on this matter).

Before examining in the next section the propagation of the errors on the kinematic variables, let us consider the other characteristic of the rescaling, i.e. the increased dependence on the electron variables, since the rescaling factor depends also on the reconstructed energy and angle of the scattered electron. We can indirectly demonstrate the increased dependence by applying a further rescaling, and obtain three new methods: $r_{2} \Sigma, r_{2} \mathrm{D} \Sigma$ and $r_{2} e \Sigma$. The $r_{2}$ rescaling factor is obtained recursively (assuming 
$r_{0}=1, r_{1}=r$ and $\left.y_{r_{0} \Sigma}=y_{\Sigma}\right)$ using the following formulae:

$$
y_{r_{n} \Sigma}=\frac{y_{r_{n-1} \Sigma}}{y_{r_{n-1} \Sigma}+1-y_{e}} \quad \text { and } \quad r_{n}=\frac{2 E_{\circ}}{r_{n-1} \Sigma+\Sigma_{e}}
$$

The resolution at high $y$ improves at each rescaling and actually $y_{r_{n} \Sigma} \rightarrow y_{e}$ when $n \rightarrow \infty$ implying that the three approaches $\left(r_{n} \Sigma, r_{n} \mathrm{D} \Sigma\right.$ and $\left.r_{n} e \Sigma\right)$ converge to the $e$ method. This means that the gain in precision at high $y$ is obtained at the expense of a loss of precision at low $y$. Furthermore the "hadronic" method becomes more influenced by the $e$ errors and the significance of the cross-check of the systematics which can be made when using the $e$ and $\Sigma$ methods independently is reduced at each rescaling. However, we will now study the influence of the hadronic and electron reconstruction errors on these methods, to see when the usage of a rescaled method is worthwile.

\section{Error Propagation on the Kinematic Variables}

In this section we will first consider that the hadronic final state reconstruction is the dominant source of systematic error on the kinematic variables, and thus neglect the effect of the electron reconstruction, which will be considered specifically in the second part of the section. This approximation is legitimate at HERA, in particular at low $Q^{2}$, since the energy carried by the hadronic final state which is visible in the detector becomes on average smaller as $Q^{2}$ decreases. Since the hadronic final state is a combination of several particles which enter the detector in different places, the precise effect of the detector response can be obtained only via a complete detector simulation/reconstruction program. However we can start by examining the propagation of the observable errors on the kinematic variables, before comparing the results to a realistic simulation. Namely we will consider errors like $\delta \Sigma / \Sigma$ and $\delta p_{T, h} / p_{T, h}$ which are related to the error on the hadronic angle $\delta \gamma$ by $\llbracket$

$$
\frac{\delta \gamma}{\sin \gamma}=\frac{\delta \Sigma}{\Sigma}-\frac{\delta p_{T, h}}{p_{T, h}}
$$

The error on $\gamma$ arises from the spread of the hadronic final state particles in the detector, since it is not possible to ensure complete homogeneity in the detector response (even with a perfect absolute calibration, and with a perfect angular reconstruction, the fluctuations in the energy measurement have a different influence on $\Sigma$ and $p_{T, h}$ as soon as there is more than one particle in the final state). In addition particle losses in the beam pipe affect $\Sigma$ and $p_{T, h}$ in a different way: losses in the forward (backward) beam pipe affect to a good approximation mainly $p_{T, h}(\Sigma)$. This explains why, at low $Q^{2}$, the DA method is not precise both at very low and very high $y$. The complete

\footnotetext{
${ }^{6}$ Since $y_{r_{n} \Sigma}$ can be written as $\frac{1}{1+u+u^{2}+. .+u^{n-1}+u^{n} / y_{\Sigma}}$, with $u \equiv 1-y_{e}$

${ }^{7}$ In all the error equations, negative signs may appear in front of some terms, allowing to know the direction of the bias introduced by a given measurement error. When the terms are independent, the actual total error squared is obtained as usual, i.e. by quadratically summing the different terms.
} 


\begin{tabular}{|c||c|c|c|c|c|}
\hline & $\Sigma$ & $\mathrm{D} \Sigma$ & $e \Sigma$ & $\mathrm{PT}(\equiv r \mathrm{D} \Sigma)$ & $r \Sigma$ \\
\hline \hline$\frac{\delta y}{y}$ & $(1-y) \frac{\delta \Sigma}{\Sigma}$ & $(1-y) \frac{\delta \Sigma}{\Sigma}$ & $(1-2 y) \frac{\delta \Sigma}{\Sigma}$ & $(1-y)\left(1-y_{r}\right) \frac{\delta \Sigma}{\Sigma}$ & $(1-y)\left(1-y_{r}\right) \frac{\delta \Sigma}{\Sigma}$ \\
\hline$\frac{\delta Q^{2}}{Q^{2}}$ & $y \frac{\delta \Sigma}{\Sigma}$ & $-y \frac{\delta \Sigma}{\Sigma}$ & 0 & $-y_{r}(1-y) \frac{\delta \Sigma}{\Sigma}$ & $y_{r}(1-y) \frac{\delta \Sigma}{\Sigma}$ \\
\hline$\frac{\delta x}{x}$ & $(2 y-1) \frac{\delta \Sigma}{\Sigma}$ & $-y \frac{\delta \Sigma}{\Sigma}$ & $(2 y-1) \frac{\delta \Sigma}{\Sigma}$ & $-\frac{\delta \Sigma}{\Sigma}$ & $\left(2 y_{r}-1\right)(1-y) \frac{\delta \Sigma}{\Sigma}$ \\
\hline
\end{tabular}

Table 2: Errors on $y, Q^{2}$ and $x$ due to errors on the hadronic observables for the $\Sigma$, $D \Sigma, e \Sigma, P T(=r D \Sigma)$ and $r \Sigma$ methods. For the coefficients in this table, the following abreviated notations are used: $\quad y \equiv y_{\Sigma} ; \quad y_{r} \equiv y_{r \Sigma}$.

error propagation of $\delta \Sigma$ and $\delta \gamma$ for $x, y$ and $Q^{2}$ for the $h$, DA and $m$ methods are given in the appendix, but are not discussed further here since these methods do not allow a precise measurement over the full kinematic range.

For the optimized and for the rescaled methods, the corresponding error propagation are given in table 2, noting that they depend only on one hadronic variable, $\Sigma$. At low $y(y \leq 0.1)$, the errors of the three methods become identical:

$$
\frac{\delta y}{y} \simeq \frac{\delta \Sigma}{\Sigma} \quad \frac{\delta Q^{2}}{Q^{2}} \simeq 0 \quad \frac{\delta x}{x} \simeq-\frac{\delta \Sigma}{\Sigma}
$$

and a more precise study of the effect of the losses in the forward beam pipe and of the $p_{T, e}$ reconstruction uncertainties is needed since they become more important than those due to the hadronic energy uncertainty.

At high $y$ the error originating from the hadrons dominates and the situation is drastically changed:

- For the optimized reconstruction methods, the $\Sigma$ approach allows smaller errors than the DA or D $\Sigma$ approach, as can be seen by comparing $\delta x_{\Sigma} / x_{\Sigma}$ to $\delta x_{D \Sigma} / x_{D \Sigma}$ in the $y$ interval $0.3-0.7$. The computed average ratio of these errors is $1 / 3$ and is even smaller if comparing the $\Sigma$ to the DA method. This explains the results already discussed of fig. 田.

- For the rescaled methods (PT and $r \Sigma$ ) the errors on $x$ and $Q^{2}$, with respect to the corresponding non-rescaled method are reduced by a factor $(1-y)$, providing an improvement at high $y$. Both methods have a reconstructed $Q^{2}$ which deviates from the true $Q^{2}$ by the same amount but in opposite directions. For $x$ we have a similar situation as for the non-rescaled case since the improvement by a factor of $(1-y)$ in both methods does not cancel the difference in resolution observed above between $x_{\Sigma}$ and $x_{D \Sigma}$, so the $r \Sigma$ (and actually also the simple $\Sigma$ ) method provides a better estimation of $x$ than the PT one. So, when the hadronic error is the dominant one, a method of the Sigma type should be chosen to have the best kinematic reconstruction over the widest kinematic range. 
As already said, the "hadronic" methods described above are also influenced by the reconstruction of the electron angle and energy. Here we examine the situation at low $Q^{2}$ in which typical values for $\delta E / E$ and $\delta \theta$ in the HERA experiments are $1 \%$ and 1 mrad. The case of high $Q^{2}$ events is treated in section 6 . From the error propagation of $\mathrm{E}$ and $\theta$ on the kinematic variables reconstructed with the $e$ method, we can observe that the $\theta$ error becomes significant only when $\theta$ tends to its extreme $\left(0\right.$ and $\left.180^{\circ}\right)$. At low $Q^{2}$, i.e. if $\theta$ is greater than about $175^{\circ}$ its error dominates the total error. For the following discussion we do not consider $\delta \theta$ if it is not weighted by a $\tan \theta / 2$ factor large enough to render this error comparable to the error originating from the electron energy miscalibration. Considering only the uncertainty due to the angular measurement, we have:

$$
\frac{\delta y_{e}}{y_{e}} \simeq 0 \quad \frac{\delta Q_{e}^{2}}{Q_{e}^{2}} \simeq-\tan \frac{\theta}{2} \delta \theta \quad \frac{\delta x_{e}}{x_{e}} \simeq-\tan \frac{\theta}{2} \delta \theta
$$

For the DA method we have a different $\theta$ dependence (see table 5):

$$
\frac{\delta y_{D A}}{y_{D A}}=-\frac{1-y_{D A}}{\sin \theta} \delta \theta \quad \frac{\delta Q_{D A}^{2}}{Q_{D A}^{2}} \simeq-\frac{2}{\sin \theta} \delta \theta \quad \frac{\delta x_{D A}}{x_{D A}} \simeq-\frac{1+y_{D A}}{\sin \theta} \delta \theta
$$

implying a more severe degradation at low $Q^{2}$ than for the $e$ method, since $y_{D A}$ is strongly affected by the $\theta$ error, and $x_{D A}, Q_{D A}^{2}$ are more affected than $x_{e}, Q_{e}^{2}$.

To study the other "hadronic" methods we observe that the errors on $\Sigma_{e}$ and $r$ due to $\delta \theta$ are such that:

$$
\delta \Sigma_{e} / \Sigma_{e} \simeq 0 \quad ; \quad \delta r / r \simeq 0
$$

implying that for all hadronic methods which use $p_{T, e}(\Sigma, \mathrm{D} \Sigma, r \Sigma, \mathrm{PT}, e \Sigma, .$.$) the errors$ due to $\theta$ (see also table 6 ) are identical, namely:

$$
\frac{\delta y}{y} \simeq 0 \quad \frac{\delta Q^{2}}{Q^{2}} \simeq-\tan \frac{\theta}{2} \delta \theta \quad \frac{\delta x}{x} \simeq-\tan \frac{\theta}{2} \delta \theta
$$

We thus have a simple situation: the error on $\theta$ propagates in the same way in the $e$ and in all these hadronic methods and this fact comes from the usage of the transverse momentum of the electron. Since $y$ is essentially not affected by the $\delta \theta$ error, similarly all hadronic $y$ are also independent of this error. However, both $Q^{2}$ and $x$ have a divergent error at large angle, implying that the measurement of the lowest $Q^{2}$ by using larger angles is hampered by the angle error propagation. This suggests the usage of lower incident (beam) electron energies to measure precisely the lower $Q^{2}$ domain, rather than further increasing the acceptance at large angle as has already been achieved at HERA, by shifting the interaction position by about $70 \mathrm{~cm}$ in the forward direction and by upgrading the detectors close to the backward beam pipe.

To study now the influence of the electron energy $\mathrm{E}$ on the standard and rescaled methods, we compare in table 3 the energy error propagation for the $\Sigma, \mathrm{D} \Sigma, e \Sigma, \mathrm{PT}$ and $r \Sigma$ methods, keeping in mind for comparison the well-known $\frac{1}{y} \frac{\delta E}{E}$ behaviour of $\delta x_{e} / x_{e}$. The lack of dependence on $\mathrm{E}$ in the DA method is changed to a weak 


\begin{tabular}{|c||c|c|c|c|c|}
\hline & $\Sigma$ & $\mathrm{D} \Sigma$ & $e \Sigma$ & $\mathrm{PT}(\equiv r \mathrm{D} \Sigma)$ & $r \Sigma$ \\
\hline \hline$\frac{\delta y}{y}$ & $(y-1) \frac{\delta E}{E}$ & $(y-1) \frac{\delta E}{E}$ & $2(y-1) \frac{\delta E}{E}$ & $\left(y_{r}-1\right)(2-y) \frac{\delta E}{E}$ & $\left(y_{r}-1\right)(2-y) \frac{\delta E}{E}$ \\
\hline$\frac{\delta Q^{2}}{Q^{2}}$ & $(2-y) \frac{\delta E}{E}$ & $y \frac{\delta E}{E}$ & $\frac{\delta E}{E}$ & $y_{r}(2-y) \frac{\delta E}{E}$ & $2-y_{r}(2-y) \frac{\delta E}{E}$ \\
\hline$\frac{\delta x}{x}$ & $(3-2 y) \frac{\delta E}{E}$ & $\frac{\delta E}{E}$ & $(3-2 y) \frac{\delta E}{E}$ & $(2-y) \frac{\delta E}{E}$ & $2-\left(2 y_{r}-1\right)(2-y) \frac{\delta E}{E}$ \\
\hline
\end{tabular}

Table 3: Errors on $y, Q^{2}$ and $x$ due to errors on the energy of the electron for the $\Sigma$, $D \Sigma, e \Sigma, P T$ and $r \Sigma$ methods. The following notations are used: $y \equiv y_{\Sigma} ; \quad y_{r} \equiv y_{r \Sigma}$.

dependence in the $\mathrm{D} \Sigma$ method. The $\mathrm{E}$ dependence of $Q^{2}$ for the $\Sigma$ method (and to a lesser extent for the $e$ method: $\delta Q_{e}^{2} / Q_{e}^{2}=\frac{\delta E}{E}$ ), is stronger than the $\mathrm{D} \Sigma$ one. At high $y$ however, the influence of $\mathrm{E}$ on the $Q^{2}$ of these three methods become similar. A similar effect is visible on $x$, for which the $\mathrm{E}$ dependence of the $\mathrm{D} \Sigma$ is small but increases at high $y$, while it is larger for the $\Sigma$ and $e$ methods at low $y$ but decreases with increasing $y$. For the rescaled methods the same message holds, but the $\mathrm{E}$ dependence on the PT method is larger than on the $\mathrm{D} \Sigma$ one (a factor $2-y$ larger, for all variables), and conversely, smaller for the $r \Sigma$ than for the $\Sigma$ one (from $\propto 2-y$ to $\propto 2-2 y$ ), thereby reducing the initial difference between the $\Sigma$ and $\mathrm{D} \Sigma$ methods.

As a general conclusion, the PT method is better suited for low $y$ than for high $y$ and is less influenced by the electron energy miscalibration. The usage of the PT method should thus be reserved to the case in which the miscalibration error on the electron is of the same order as the hadronic one. This can be verified by comparing fig. Ta, which shows the purity at $Q^{2}=20 \mathrm{GeV}^{2}$ of the $e \Sigma$ and of the three rescaled methods in the nominal calibration conditions of the H1 experiment, to fig. $7 \mathrm{~b}$, which
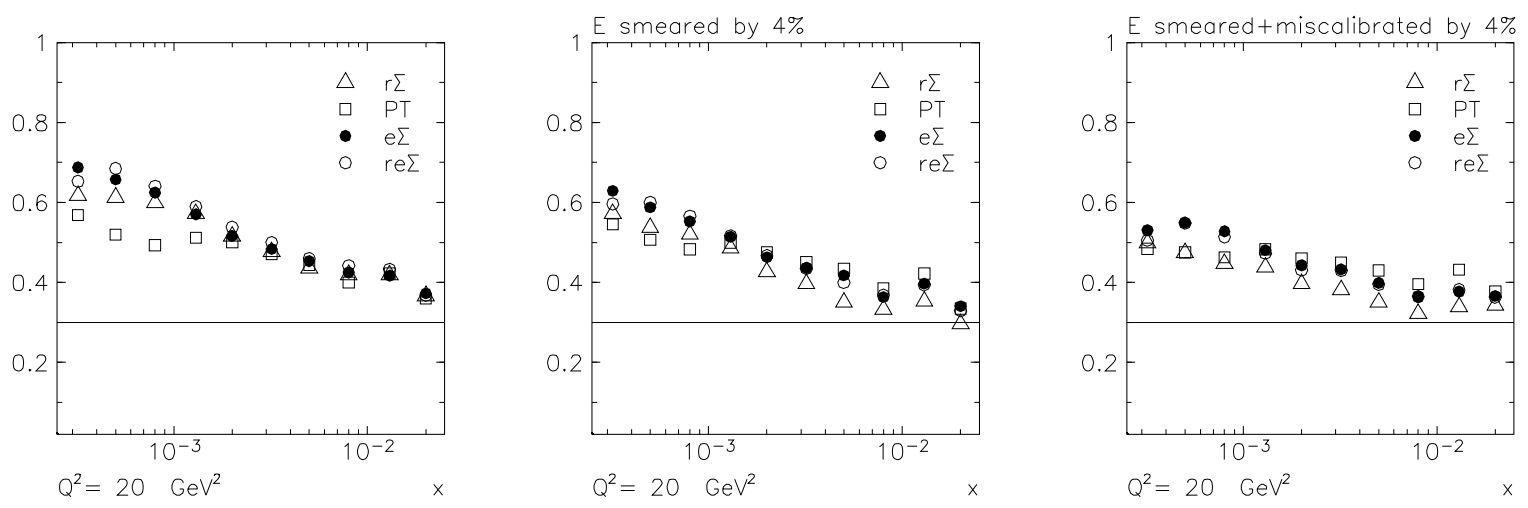

Figure 7: Purity at $Q^{2}=20 \mathrm{GeV}^{2}$ for 4 kinematic reconstruction methods: $r \Sigma$ (open triangles), PT (open squares), $e \Sigma$ (closed circles) and re $\Sigma$ (open circles). a) nominal calibration; b) Electron energy smeared by 4\%; c) Electron energy smeared and miscalibrated by $4 \%$. 
shows the same purities, but obtained after degradation of the electron energy response with a gaussian smearing of $4 \%$, and to fig. $7 \mathrm{c}$, which has an additional systematic shift on the electron energy scale of $-4 \%$. The figures show how the PT method remains relatively insensitive to the energy degradation, thereby becoming more precise than the other methods which are more affected by the degradation.

\section{$6 \quad$ Kinematics at High $Q^{2}$}

With the foreseen increase of luminosity, the HERA physics program in the next decade will focus on physics at large momentum transfer. These studies have been already started, and have been spurred by the observation of an excess of NC DIS events at high $Q^{2}\left(Q^{2}>15000 \mathrm{GeV}^{2}\right)$ 23, 24]. The difficulty of reconciling both the $\mathrm{H} 1$ and ZEUS signals [2, 25] in the hypothesis of a single narrow resonance has underlined the importance of kinematic reconstruction at high $Q^{2}$ in particular when dealing with low statistics on which radiative effects can have a strong influence. For the future data, a simple comparison of the different methods can be made, based on the bin purities and using high simulated statistics. A more elaborate study would require for instance to study the optimal $\left(x, Q^{2}\right)$ binning for each method. However the basic properties can be understood with a single binning choice well adapted to the goal pursued by the two collaborations, i.e. an uncertainty on the electron and hadronic energy measurement of about 1 and $2 \%$ respectively.

We compare in selected high $Q^{2}$ bins, in fig. 8, the optimal $e \Sigma$ method to the two simple methods which are precise at high $Q^{2}$, i.e. the $e$ and DA ones. The DA method has a good purity in the whole $\left(x, Q^{2}\right)$ range displayed, but is in general less precise than the $e$ or $e \Sigma$ method. These two last methods display a similar behaviour at high $Q^{2}$, both at high and low $x$. At $Q^{2}$ below $10000 \mathrm{GeV}^{2}$, the low $y$ weakness of the $e$ method starts to be visible in the highest $x$ points (see also fig. 2).
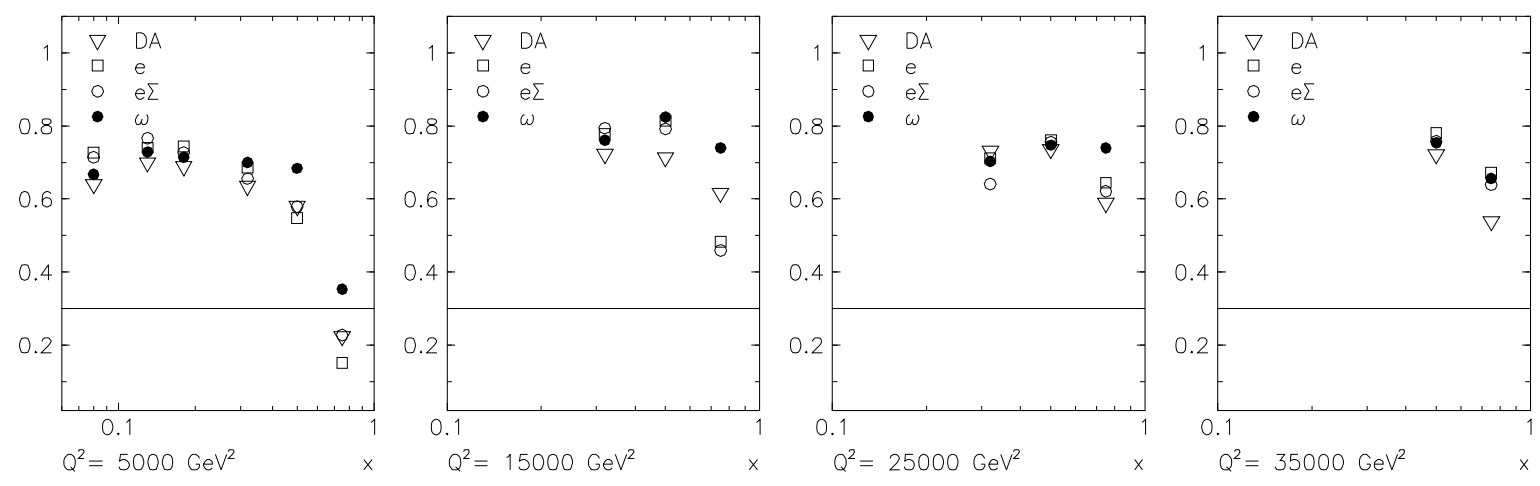

Figure 8: Purity at high $Q^{2}$ for 4 kinematic reconstruction methods: e (open squares), $D A$ (open triangles), $e \Sigma$ (open circles) and $\omega$ (closed circles). 
These three methods are also compared to the $\omega$ method which was introduced in [2] as an extension of the $\Sigma$ method in which the hadronic final state response is recalibrated using energy-momentum conservation, and assuming that the hadronic miscalibration effects propagate in a similar way in $p_{T, h}$ and $\Sigma$, i.e $\delta p_{T, h} / p_{T, h} \simeq \delta \Sigma / \Sigma$. This approximation is correct at $Q^{2}$ above a few thousands $\mathrm{GeV}^{2}$. For instance, the resolution of the inclusive invariant mass $\mathrm{M}_{\omega}$, which is related to $x_{\omega}$ via $\mathrm{M}=\sqrt{x \cdot s}$, was shown to be excellent at high $Q^{2}$. It can be seen in fig. 8, how this method is superior to the others at high $x$, while staying comparable to the best one at low $x$. Using $Q_{e}^{2}$ instead of $Q_{\omega}^{2}$ in the $\omega$ method (i.e. an "ew" method, not shown) further improves its purity by about $5 \%$ in all bins, rendering it optimal in all these high $Q^{2}$ bins.

More generally, it can be noticed that the stability and purity are significantly higher at high $Q^{2}$ than at low $Q^{2}$, thus a finer binning both in $x$ and $Q^{2}$ could be used provided the recorded number of events is large enough. In this respect, the high luminosity HERA upgrade is indeed a very challenging program and will allow to explore in fine detail the quark-gluon structure of the nucleon at the highest $Q^{2}$. These studies will also make use of the charged current events, for which the kinematics, which has not been studied here, also improves at high $Q^{2}$ (see for instance [14]). This is due to the improved precision on $p_{T, h}$, itself coming from the stronger collimation and higher energy of the jet(s).

\section{Conclusion}

After a brief sketch of some experimental problems encountered in extracting the structure function $F_{2}$ from the measured cross-sections, we have reviewed and compared in detail the different ways of reconstructing at HERA the kinematic variables of the inclusive deep inelastic scattering. At low $x$, the effect of rescaling the hadronic energy using both hadronic and electron observables has been studied and generalized to all methods, allowing one to derive new properties intermediate between the $e$ and the $\Sigma$ methods. The error propagations allow one to choose the most sensible method depending on the kinematic region under study and on the available precision of the energy and hadronic calibration. The PT method has been compared to the $e, \Sigma$, $r \Sigma$ and $e \Sigma$ method and is found to be well suited for low $y$ but less precise than the other methods at high $y$ (i.e. low $x$ ) if the electron energy reconstruction is precise $(\delta \mathrm{E} / \mathrm{E} \simeq 1-2 \%)$. In this case the $e \Sigma$ is the most precise method over the full kinematic range. At high $Q^{2}$ it has been shown that the kinematic reconstruction becomes very precise and that further optimization is obtained by using the complete information coming from the electron and from the hadronic system, as it is done for instance in the $\omega$ method. This potential precision underlines the necessity to have high statistics

to reach a systematic limitation on the measurement and renders the exploration of the dynamics of the high $Q^{2}$ collisions, which will be done with the HERA luminosity upgrade program, particularly exciting. 


\section{Acknowledgments}

This work has taken place within the H1 collaboration, and thus has benefitted from the common efforts of all our colleagues and we would like to thank them warmly. In particular we thank Beatriz Gonzalez-Pineiro for the common work we did in the 1994 structure function analysis in which we started the studies introduced in section 2. Our ZEUS colleagues involved in the structure function measurements are also thanked for their friendly competition. We also would like to thank John Dainton, Robin Devenish, Ralph Eichler, and Jörg Gayler for a careful reading of the manuscript and for their useful remarks.

\section{References}

[1] Yu. L. Dokshitzer, Sov. Phys. JETP 46 (1977) 641;

V. N. Gribov and L.N. Lipatov, Sov. J. Nucl. Phys. 15 (1972) 438 and 675;

G. Altarelli and G. Parisi, Nucl. Phys. B126 (1977) 297.

[2] U. Bassler and G. Bernardi, Z.Phys. C76 (1997) 223

[3] G. Ingelman and R. Rückl, Proceedings of the Workshop Physics at HERA, vol. 1, eds. W. Buchmüller, G. Ingelman, DESY (1992) 107

[4] V. Blobel, DESY 84/118 (1984)

[5] M. Virchaux, Ph.D. thesis, University of Paris VII, 1988, unpublished.

[6] G. Zech, DESY 95/113 (1995)

[7] ZEUS Collab., M. Derrick et al., Z. Phys. C72 (1996) 399.

[8] B. Gonzalez-Pineiro, Ph.D. thesis, University of Paris XI, 1996, unpublished.

L. Schoeffel, Ph.D. thesis, University of Paris XI, 1997, unpublished.

[9] H1 Collab., S. Aid et al., Nucl. Phys. B470 (1996) 3.

[10] S. Bentvelsen et al., Proceedings of the Workshop Physics at HERA, vol. 1, eds. W.Buchmüller, G.Ingelman, DESY (1992) 23-40.

C.Hoeger, ibid., 43-55.

[11] U. Bassler and G. Bernardi, Nucl. Instr. and Meth. A361 (1995) 197.

[12] A. Blondel and F. Jacquet, Proceedings of the Study of an ep Facility for Europe, ed. U.Amaldi, DESY 79/48 (1979) 391

[13] J. Blümlein and M. Klein, Proceedings of the Snowmass Workshop "The Physics of the Next Decade", ed. R. Craven, (1990) 549. 
[14] G.Bernardi and W.Hildesheim, Proceedings of the Workshop Physics at HERA, vol. 1, eds. W. Buchmüller, G. Ingelman, DESY (1992) 79

[15] H1 Collab., I. Abt et al., Nucl. Phys. B407 (1993) 515

[16] H1 Collab., C. Adloff et al., DESY 97-158 (1997), submitted to Z. Phys. C

[17] A. Mehta, private communication.

[18] H1 Collab., C. Adloff et al., DESY 97-042 (1997) submitted to Nucl. Phys. B.

[19] H1 Collab., C. Adloff et al., A Measurement of the Inclusive Deep Inelastic ep Scattering Cross-Section at low $Q^{2}$ at HERA, contributed paper submitted to EPS 97, Jerusalem (1997).

[20] H1 Collab., C. Adloff et al., A Measurement of the Proton Structure Function $F_{2}\left(x, Q^{2}\right)$ at High $Q^{2}$ at HERA, contributed paper submitted to EPS 97, Jerusalem (1997).

[21] H. Chaves, R.J. Seyfert and G. Zech, Proceedings of the Workshop Physics at HERA, vol. 1, eds. W.Buchmüller, G.Ingelman, DESY (1992) 57

[22] J.P. Phillips, Manchester preprint MAN/HEP/93/9 (1993), H1-note 09/93-314 (1993)

[23] H1 Collab., C. Adloff et al., Z. Phys C74 (1997) 191.

[24] ZEUS Collab., J. Breitweg et al., Z. Phys. C74 (1997) 207.

[25] M. Drees, hep-ph/9703332, APCTP 97-03 (1997) 


\section{Appendix:}

\begin{tabular}{|c|c|c|c|}
\hline method & $y$ & & $Q^{2}$ \\
\hline$e$ & $1-\frac{\Sigma_{e}}{2 E_{\circ}}$ & & $\frac{p_{T, e}^{2}}{1-y_{e}}$ \\
\hline$h$ & $\frac{\Sigma}{2 E_{\circ}}$ & & $\frac{p_{T, h}^{2}}{1-y_{h}}$ \\
\hline DA & $\frac{\tan \gamma / 2}{\tan \gamma / 2+\tan \theta / 2}$ & $4 \mathrm{E}_{\circ}^{2}$ & $\frac{\cot \theta / 2}{\tan \gamma / 2+\tan \theta / 2}$ \\
\hline$m$ & $y_{h}$ & & $Q_{e}^{2}$ \\
\hline$\Sigma$ & $\frac{\Sigma}{\Sigma+\Sigma_{e}}$ & & $\frac{p_{T, e}^{2}}{1-y_{\Sigma}}$ \\
\hline $\mathrm{D} \Sigma$ & $y_{D \Sigma}=y_{\Sigma}$ & $4 \mathrm{E}_{\circ}^{2}$ & $\frac{\cot \theta / 2}{\tan \gamma_{\Sigma} / 2+\tan \theta / 2}$ \\
\hline$e \Sigma$ & $y_{e \Sigma}=r y_{\Sigma}$ & & $Q_{e}^{2}$ \\
\hline$r \Sigma$ & $\frac{r \Sigma}{r \Sigma+\Sigma_{e}}$ & & $\frac{p_{T, e}^{2}}{1-y_{r \Sigma}}$ \\
\hline $\mathrm{PT}(\equiv r \mathrm{D} \Sigma)$ & $y_{P T}=y_{r \Sigma}$ & $4 \mathrm{E}_{\circ}^{2}$ & $\frac{\cot \theta / 2}{\tan \gamma_{r \Sigma} / 2+\tan \theta / 2}$ \\
\hline re $\Sigma$ & $y_{r e \Sigma}=r_{2} y_{r \Sigma}$ & & $Q_{e}^{2}$ \\
\hline
\end{tabular}

Table 4: $y$ and $Q^{2}$ formulae for the electron and the main hadronic methods discussed in the text, using: $\Sigma \equiv \Sigma_{h}\left(E_{h}-p_{z, h}\right), \Sigma_{e} \equiv E(1-\cos \theta), r \equiv \frac{2 E_{\circ}}{\Sigma+\Sigma_{e}}$ and $r_{2} \equiv \frac{2 E_{\circ}}{r \Sigma+\Sigma_{e}}$ 


\begin{tabular}{|c|c|c|c|}
\hline & $h$ & DA & $m$ \\
\hline$\frac{\delta y}{y}$ & $\frac{\delta \Sigma}{\Sigma}$ & $\frac{1-y_{D A}}{\sin \gamma} \delta \gamma-\frac{1-y_{D A}}{\sin \theta} \delta \theta$ & $\frac{\delta \Sigma}{\Sigma}$ \\
\hline$\frac{\delta Q^{2}}{Q^{2}}$ & $2 \frac{\delta p_{T, h}}{p_{T, h}}+\frac{y_{h}}{1-y_{h}} \frac{\delta \Sigma}{\Sigma}$ & $-\frac{y_{D A}}{\sin \gamma} \delta \gamma-\left(\frac{1}{\sin \theta}+\frac{y_{D A}}{2 \cos ^{2} \frac{\theta}{2} \tan \frac{\gamma}{2}}\right) \quad \delta \theta$ & $\frac{\delta E}{E}-\tan \frac{\theta}{2} \delta \theta$ \\
\hline$\frac{\delta x}{x}$ & $2 \frac{\delta p_{T, h}}{p_{T, h}}+\frac{2 y_{h}-1}{1-y_{h}} \frac{\delta \Sigma}{\Sigma}$ & $-\frac{1}{\sin \gamma} \delta \gamma-\left(\frac{y_{D A}}{\sin \theta}+\frac{y_{D A}}{2 \cos ^{2} \frac{\theta}{2} \tan \frac{\gamma}{2}}\right) \quad \delta \theta$ & $\frac{\delta E}{E}-\tan \frac{\theta}{2} \delta \theta-\frac{\delta \Sigma}{\Sigma}$ \\
\hline
\end{tabular}

Table 5: Errors on $y, Q^{2}$ and $x$ originating from the errors on the measured variables $\Sigma, p_{T, h}, \gamma, E$, and $\theta$, for the $h, D A$ and $m$ methods.

\begin{tabular}{|c|c|c|c|c|}
\hline & $e$ & $\Sigma$ & $\mathrm{D} \Sigma$ & $\mathrm{PT}(\equiv r \mathrm{D} \Sigma)$ \\
\hline$\frac{\delta y}{y} \frac{1}{\delta \theta}$ & $\frac{1}{y_{e}} \frac{y_{e}-1}{\tan \frac{\theta}{2}}$ & $\frac{y_{\Sigma}-1}{\tan \frac{\theta}{2}}$ & $\frac{y_{\Sigma}-1}{\tan \frac{\theta}{2}}$ & $\frac{\left(2-y_{\Sigma}\right)\left(y_{r \Sigma}-1\right)}{\tan \frac{\theta}{2}}$ \\
\hline$\frac{\delta Q^{2}}{Q^{2}} \frac{1}{\delta \theta}$ & $-\tan \frac{\theta}{2}$ & $-\tan \frac{\theta}{2}+\frac{1-y_{\Sigma}}{\tan \frac{\theta}{2}}$ & $-\tan \frac{\theta}{2}-\frac{1-y_{\Sigma}}{\tan \frac{\theta}{2}}$ & $-\tan \frac{\theta}{2}+\frac{\left(2-y_{\Sigma}\right) y_{r \Sigma}}{\tan \frac{\theta}{2}}$ \\
\hline$\frac{\delta x}{x} \frac{1}{\delta \theta}$ & $-\tan \frac{\theta}{2}-\frac{1}{y_{e}} \frac{y_{e}-1}{\tan \frac{\theta}{2}}$ & $-\tan \frac{\theta}{2}+\frac{2\left(1-y_{\Sigma}\right)}{\tan \frac{\theta}{2}}$ & $-\tan \frac{\theta}{2}$ & $-\tan \frac{\theta}{2}+\frac{\left(2-y_{\Sigma}\right)}{\tan \frac{\theta}{2}}$ \\
\hline
\end{tabular}

Table 6: Errors on $y, Q^{2}$ and $x$ due to the error on the polar angle of the electron for the e, $\Sigma, D \Sigma$ and PT methods. In the $e \Sigma$ method, $\frac{\delta y}{y}$ is two times larger than for the $\Sigma$ method, i.e. $\frac{\delta y}{y}=2 \frac{y_{\Sigma}-1}{\tan \frac{\theta}{2}} \delta \theta$. In the $r \Sigma$ method: $\frac{\delta Q^{2}}{Q^{2}}=-\tan \frac{\theta}{2}+\frac{2-y_{r \Sigma}\left(2-y_{\Sigma}\right)}{\tan \frac{\theta}{2}} \delta \theta$. 

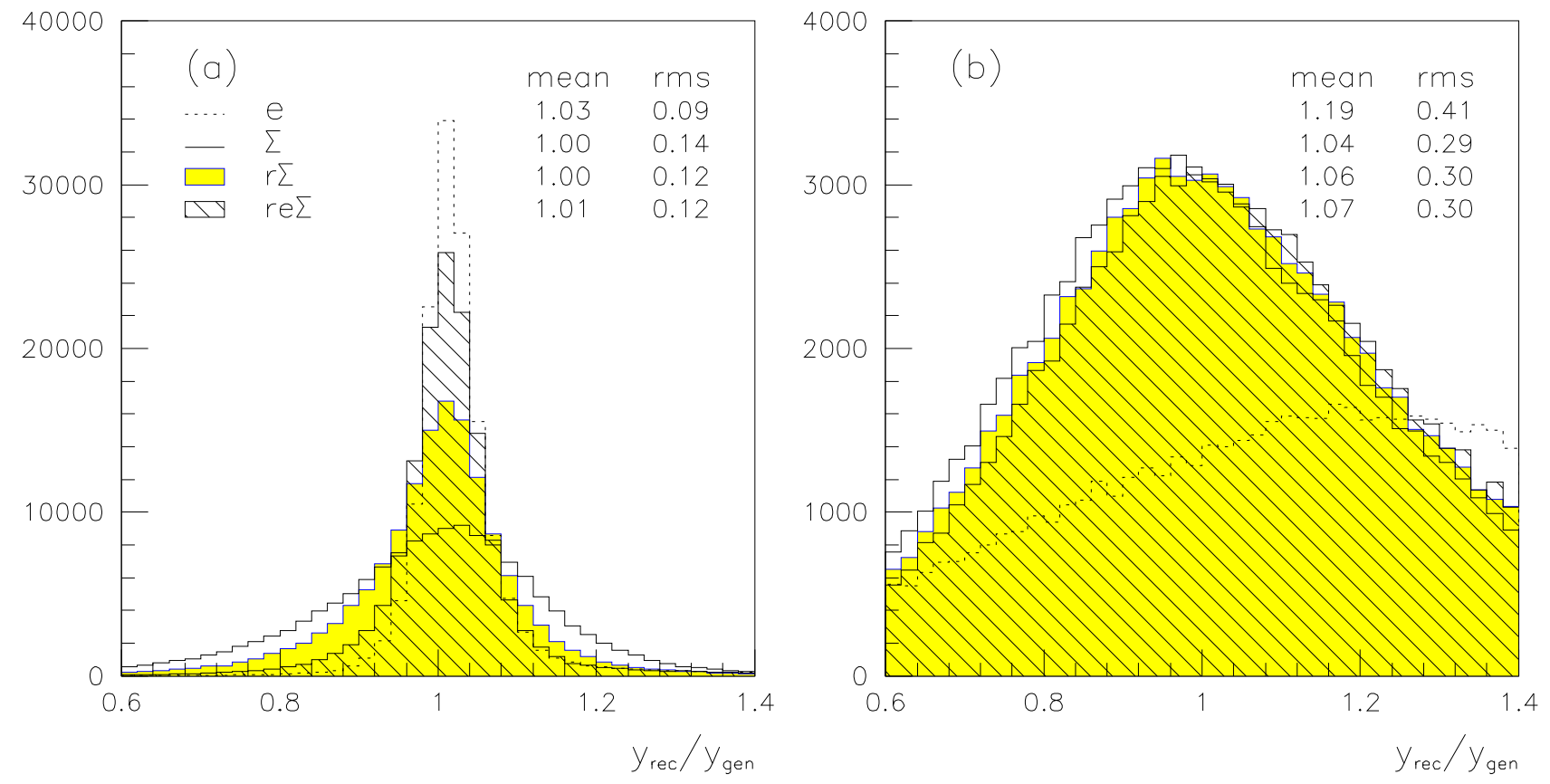